\title{
ANÁLISE DA DISPOSIÇÃO DE CONSUMO POR ORGÂNICOS EM UMA INDÚSTRIA DE CEREAIS ANALYSIS OF CONSUMPTION DISPOSAL BY ORGANIC IN A CEREAL INDUSTRY
}

\author{
Pang Lien Hsu ${ }^{1}$ \\ Emerson Dietrichkeit ${ }^{2}$ \\ José Vinícius Santos Barboza ${ }^{3}$ \\ Jerry Adriani Johann ${ }^{4}$ \\ Geysler Rogis Flor Bertolini ${ }^{5}$
}

\section{Resumo}

O artigo apresentado tem por objetivo analisar se os consumidores estão dispostos e até quanto pagariam para adquirir produtos orgânicos em relação aos produtos tradicionais de uma indústria de cereais do interior do Paraná, sendo escolhidos para o trabalho, os três mais vendidos pela organização: Milho para pipoca, Amendoim e Canjiquinha. O estudo em questão foi aplicado a 221 consumidores em dois supermercados localizados em Cascavel - PR e os resultados foram analisados pelo modelo Bertolini (2009) adaptado. O estudo demonstrou que há consumidores dispostos a pagarem um valor adicional por produtos orgânicos, como também gerou um estudo preliminar em relação aos custos e retornos que a empresa pode sofrer com a oferta dos produtos orgânicos, demonstrando que podem comercializar esses produtos no mercado a um custo unitário superior ao tradicional, além de que foi possível identificar que os consumidores que não pagariam a mais pelo produto orgânico continuariam a consumir o produto tradicional.

Palavras-chave: Produtos Orgânicos. Disposição a pagar. Comportamento do Consumidor.

1 Doutor em Administração pela Universidade Nove de Julho - UNINOVE. São Paulo, São Paulo, Brasil. E-mail: panghsu@hotmail.com

2 Mestre em Administração pela Universidade Estadual do Oeste do Paraná - UNIOESTE. Engenheiro eletricista consultor da Companhia Paranaense de Energia - Copel, Cascavel, Paraná, Brasil. E-mail: emerson.dietrichkeit@hotmail. com

3 Mestre em Administração pela Universidade Estadual do Oeste do Paraná - UNIOESTE. Assistente de Negócios do Banco do Brasil S.A., Cascavel, Paraná, Brasil. E-mail: josevinicius@ hotmail.com

4 Doutor em Engenharia Agrícola pela Universidade Estadual de Campinas - UNICAMP. Docente na Universidade Estadual do Oeste do Paraná - UNIOESTE, Cascavel, Paraná, Brasil. E-mail: jerry.johann@ @otmail.com

5 Doutor em Engenharia de Produção pela Universidade Federal de Santa Catarina - UFSC. Docente na Universidade Estadual do Oeste do Paraná - UNIOESTE, Cascavel, Paraná, Brasil. E-mail: geysler_rogis@yahoo.com.br 


\section{Abstract}

The presented article aims to examine whether consumers are willing to pay and how much they are willing to expend for organic products over traditional products of a cereal industry of Paraná state, for this case the three most sold produtcs by the organization was chosen: Maize popcorn, Peanuts and ground corn grits. The study in question was administered to 221 consumers in two supermarkets located in Cascavel - PR and the results were analyzed by adapted Bertolini model (2009). The study showed that there are consumers willing to pay an additional amount for organic products, but also generated a preliminary study on the costs and returns that the company may suffer by offering these organic products, demonstrating that they can offer these products to the market with a higher unit cost than the traditional, and also it was possible to identify that consumers who would not pay more for organic products continue to consume traditional product.

Keywords: Organic Products. Willingness to Pay. Consumer Behavior.

\section{INTRODUÇÃO}

Uma mudança vem ocorrendo na relação empresa e consumidor. Antes as organizações buscavam atingir uma grande produção a fim do lucro. Hoje os consumidores estão mais críticos e possuem um maior poder de barganha na hora de escolher o que consumir. As empresas para se manterem competitivas estão se adaptando e mudando para atender esse novo modelo de mercado que cobra das organizações atitudes proativas em relação a questões ambientais. Sendo assim a imagem de uma organização está relacionada a forma de produção e ações que tomam, o que pode ser tanto uma ameaça como uma oportunidade.

Independentemente de como a organização definirá sua estratégia, é possível utilizar esta nova postura para se promover no mercado, mostrando aos consumidores que valorizam a oferta de produtos ecologicamente corretos que a empresa se preocupa com o meio ambiente. Porém, para cada possibilidade de cuidados com o meio ambiente, a empresa necessita de recursos financeiros para viabilizar o projeto. $\mathrm{O}$ investimento é necessário, pois, para cada mudança na organização, existem custos para a adequação das atividades. (BERTOLINI; LEZANA; ROJO, 2012).

Pesquisas apontam a necessidade de identificar a disposição a pagar por produtos ou serviços ecológicos (TAMBOSI et al., 2014; CERDA URRUTIA, et al., 2011; ALVES; ARAÚJO; SILVA, 2017), tanto para o desenvolvimento de novos produtos (SILVA et al., 2019; BERTOLINI et al., 2013), quanto para o conhecimento da possibilidade de compra (TREVIZAN; CASEMIRO, 2009; ROMEIRO, 2012).

Diante do exposto, questiona-se o: Os consumidores estão dispostos a pagar um valor superior por produtos orgânicos de uma indústria do setor alimentício de cereais? Assim, este trabalho apresenta um modelo de análise de investimentos, adaptado de Bertolini (2009), para a tornar os produtos tradicionais de uma indústria em produtos com alguma característica ecológica. A pesquisa aplicada justifica-se em analisar a viabilidade pela empresa, levando em consideração de que alguns consumidores analisam apenas o fator preço na hora da escolha e da compra de determinado produto.

Após determinar o problema da pesquisa, o artigo identifica a percepção dos consumidores em relação ao consumo dos produtos orgânicos, até que valor pagariam para consumir esses produtos, prevê o custo do investimento e analisa a viabilidade para a produção dos produtos de forma orgânica. 


\section{AGRICULTURA SUSTENTÁVEL}

O conceito de sustentabilidade começou-se a ser discutida a partir do início do século XX, ou seja, não são conceitos novos, porém foi depois da segunda guerra mundial é que se começou a ter uma consciência maior sobre o tema, quando visões mais fortes sobre o meio ambiente e o desenvolvimento econômico atingiu um público mais amplo, pois iniciou-se a perceber que o planeta não consegue fornecer recursos em quantidades ilimitadas, somado a isso, os avanços tecnológicos, por mais que aumentaram a eficiência até aquele ponto, seria incapaz de satisfazer as demandas exponenciais de produtos, principalmente os que dependem altamente de recursos não-renováveis. (AGUIAR; CALEMAN, 2013).

Aguiar \& Caleman (2013) cita que companhias, agências públicas, organizações não-governamentais (ONGs) e todos os indivíduos estão de alguma maneira envolvidas na discussão de como promover e desenvolver sistemas sustentáveis, sendo assim, um problema que engloba a todos que também é complexa, pois além de lidar com as dimensões interdependentes - social, econômica e ambiental - eles não são contidos por limites geográficas, mas que as discussões sobre a sustentabilidade podem ser descritas como aqueles que possuem um escopo focado na qual práticas sustentáveis são discutidas dentro das empresas e/ou em pontos de vista individuais, no entanto, é necessário ter uma abordagem mais sistêmica.

No setor da agricultura não é diferente, Tilman et al.(2002) definem a agricultura sustentável como aquela em que as práticas atingem necessidades por comida e fibra das sociedades atuais e futuras, por serviços dos ecossistemas e por vidas saudáveis, e que atinge esses objetivos a partir da maximização dos benefícios líquidos para a sociedade quando todos os custos e benefícios das práticas são considerados. Os autores ainda sugerem que os impactos ambientais das práticas da agricultura são custos que geralmente não são medidos e frequentemente não influenciam o produtor nem as escolhas da sociedade nos métodos de produção. Tais custos levantam questões sobre a sustentabilidade da agricultura, dessa forma, para que a sociedade possa maximizar os benefícios líquidos da agricultura, é necessária uma contabilização mais completa tanto dos custos quando dos benefícios de práticas agrícolas alternativas, e essa contabilização deve se tornar a base para políticas, éticas e ações.

O desenvolvimento de uma agricultura sustentável deve ser acompanhado de avanços de sustentabilidade do uso da energia, manufatura, transporte e outros setores econômicos que também possuem impactos ambientais. (TILMAN et al., 2002).

Segundo Heinzen et al. (2011), uma organização inovadora sustentável não é a que introduz novidades de qualquer tipo, mas novidades que atendam as múltiplas dimensões da sustentabilidade em bases sistemáticas e colham resultados positivos para ela, para a sociedade e o meio ambiente. Não basta apenas as organizações inovarem constantemente, mas inovarem levando em consideração as três dimensões da sustentabilidade:

- Dimensão social: preocupação com os impactos sociais das inovações nas comunidades humanas dentro e fora da organização (desemprego; exclusão social; pobreza; diversidade organizacional etc.);

- Dimensão ambiental: preocupação com os impactos ambientais pelo uso de recursos naturais e pelas emissões de poluentes;

- Dimensão econômica: preocupação com a eficiência econômica, sem a qual elas não se desenvolveriam. Para as empresas essa dimensão significa obtenção de lucro e geração de vantagens competitivas nos mercados onde atuam. (HEINZEN et al., 2011).

Aguiar \& Caleman (2013) ainda citam que, apesar de vários argumentos em favor ou contra a noção de sustentabilidade, o conceito da sustentabilidade se tornou vítima por causa do equilíbrio que precisa ser mantido sobre os fatores que impactam a sustentabilidade, ou seja, o pensamento agrônomo de produtividade, o pensamento econômico do preço, pensamento sociológico da igualdade social, 
formuladores de políticas na facilitação de regras e regulações, ativistas na confrontação e processadores de alimentos no lucro.

De qualquer forma, ser mais verde e diminuir a pegada de carbono (Carbon Footprint) a partir de técnicas agrícolas que gerenciam melhor o uso dos recursos naturais, que preserve a biodiversidade e aumente a fertilidade do solo é certamente bem-vinda. A abordagem do triple bottom line (as três dimensões - social, ambiental e econômica) para a sustentabilidade têm ajudado a indústria a achar outras formas de usar os ingredientes nos produtos. (AGUIAR; CALEMAN, 2013).

\section{PRODUTOS ORGÂNICOS E O VALOR PARA OS CONSUMIDORES}

Por definição produtos orgânicos não são apenas aqueles produzidos sem a utilização de agrotóxicos, mas sim aqueles que são produzidos através de uma nova concepção, que visa harmonizar a produção agrícola com o uso racional dos recursos naturais (água, plantas, animais, etc.). Segundo Sampaio, Gosling, Fagundes \& Sousa (2013), o alimento orgânico valoriza o preparo e rotação de culturas sem o uso de produtos químicos e o seu cultivo atende a normas de certificação de qualidade. Um aspecto menos mencionado, porém não menos importante, é que na produção orgânica, os empregadores devem atender a todos os direitos trabalhistas, sendo proibido o uso de trabalho infantil.

Normalmente é classificado como um "produto verde", devido ao respeito destinado ao meio ambiente, porém ainda existe falta de informação por parte dos consumidores a respeito do conceito envolvido. Para que um produto receba a denominação de orgânico, segundo a Instrução Normativa n 46, de 6 de outubro de 2011, publicado pelo Ministério da Agricultura, Pecuária e Abastecimento, ele "deverá ser proveniente de um sistema de produção onde tenham sido aplicados os princípios e normas estabelecidos na regulamentação orgânica, por um período variável de acordo com:

I - A espécie cultivada ou manejada;

II - A utilização anterior da unidade de produção;

III - A situação ecológica atual;

IV - A capacitação em produção dos agentes envolvidos no processo produtivo; e

V - As análises e as avaliações das unidades de produção pelos respectivos organismos de avaliação de conformidade orgânica e organização de controle social”.

Foco de estudos desde a década de 90, quando surgiu como um processo alternativo que visa a promoção do desenvolvimento econômico, segundo Sampaio et al. (2013) as características dos produtos orgânicos têm despertado interesse de pesquisa em várias áreas do conhecimento, como na Nutrição e Medicina alternativa, Agricultura, Educação e Gestão ambiental. Como as regiões desenvolvidas apresentam maior consumo de orgânicos, a quantidade e localização das pesquisas envolvidas no Brasil seguem esta tendência, com mais de $75 \%$ das publicações em periódicos sobre o tema se concentrando nas regiões Sul e Sudeste do Brasil.

Nos últimos anos, a procura por alimentos orgânicos tem sido maior do que a capacidade de produção, o que tem elevado o preço de alguns produtos. De acordo com Lombardi, Moori \& Sato (2004), o fator preço caracteriza-se como o reflexo do valor que o consumidor assume para o produto.

Atualmente, segundo Ceschim \& Marchetti (2009), uma tendência muito forte da área de marketing é o estudo do comportamento do consumidor de alimentos orgânicos. Em estudo sobre o perfil do consumidor brasileiro de produtos orgânicos sinaliza que os mesmos são socialmente mais interessados, possuem a saúde como fator primordial e também se preocupam com a preservação do meio ambiente. (FREITAS et al., 2005). Segundo Trevizan \& Casemiro (2009), os consumidores são mais sensíveis às dicas e orientações de consumo provenientes de nutricionistas, médicos, professores e ambientalistas. 
Os alimentos orgânicos são reconhecidamente mais nutritivos e saudáveis, porém o seu preço é o principal impeditivo para que o mercado se expanda. Segundo Rossetti (2003), os alimentos quando básicos apresentam pouca elasticidade à renda, porém quando com maior valor agregado (como no caso dos orgânicos) são mais elásticos à renda. Para as famílias com rendas superiores, os preços dos alimentos possuem uma relevância proporcional menor do que para as famílias com rendas inferiores.

Para Lopes (2014), os consumidores apresentam percepções diferentes em relação ao valor agregado dos produtos, devido a influência da sua cultura, experiência e valores. O autor cita a definição de Calomarde (2000) de que tais características resultam em opiniões as quais influenciam o consumidor a tomar a decisão da compra de um determinado produto que possua benefícios com características ecológicas.

Aguiar \& Caleman (2013) ainda argumentam que os consumidores podem ser bastante conscientes sobre a sustentabilidade, mas não agir sustentável, de forma que ser verde, sustentável, investir em tecnologias limpas, reciclar parecem estar ao redor e que está sendo bom para as indústrias como um exercício de reduzir seus custos, porém, há de se perguntar se a pessoa está fazendo o suficiente para se tornar sustentável, pois essa consciência normalmente está concentrada no somente no nível doméstico e fortemente relacionado a atitudes para poupar dinheiro, dessa forma, sustentabilidade ainda não está fazendo com que essa consciência se traduza em padrões de consumo.

Bertolini et al. (2013) definem que quanto mais reduzido as informações dos consumidores em relação as questões ambientais, menor é a influência no interesse sobre o assunto, o que não os torna consumidores ecológicos completos, possuindo assim menor valor pelos produtos ecológicos. Estes consumidores geralmente estão dispostos a comprar produtos ecológicos, desde que o preço não seja mais elevado que os produtos convencionais, demonstrado no Quadro 1.

Quadro 1 - Parâmetros de relacionamento do valor dos consumidores com as adequações nos produtos das organizações.

\begin{tabular}{|c|c|c|}
\hline $\begin{array}{c}\text { Valor } \\
\text { para os } \\
\text { consumi- } \\
\text { dores }\end{array}$ & $\begin{array}{c}\text { Relação com o } \\
\text { preço do produ- } \\
\text { to ecológico }\end{array}$ & Expectativas em relação aos produtos ecológicos \\
\hline Alto & $\begin{array}{c}\text { Pagam mais caro } \\
\text { por um produto } \\
\text { que não agride o } \\
\text { meio ambiente. }\end{array}$ & $\begin{array}{c}\text { Produtos e embalagens fabricados com materiais reciclados e que possam } \\
\text { ser reaproveitados, produtos biodegradáveis, orgânicos e que consumam } \\
\text { menos energia ou água. E que os fabricantes possuam ações ambientais } \\
\text { proativas e certificações ambientais. }\end{array}$ \\
\hline $\begin{array}{c}\text { Modera- } \\
\text { do }\end{array}$ & $\begin{array}{c}\text { Compram os pro- } \\
\text { dutos desde que } \\
\text { possuam o mes- } \\
\text { mo preço que os } \\
\text { demais. }\end{array}$ & $\begin{array}{c}\text { Produtos que utilizem material reciclável, produtos biodegradáveis, orgâ- } \\
\text { nicos, e os que podem consumir menos energia ou água. Algumas vezes } \\
\text { podem esperar que os fabricantes possuam ações ambientais. }\end{array}$ \\
\hline Baixo & $\begin{array}{c}\text { Compram os } \\
\text { produtos se os } \\
\text { preços estiverem } \\
\text { menores que os } \\
\text { dos concorrentes. }\end{array}$ & $\begin{array}{r}\text { Produtos de material reciclado, produtos orgânicos e aqueles que podem } \\
\text { ser reaproveitados suas embalagens ou recipientes. }\end{array}$ \\
\hline
\end{tabular}

Fonte: Adaptado de Bertolini et al., (2013).

Para Heinzen et al. (2011), as inovações tecnológicas de produto e de processo podem variar de acordo com a importância atribuída à gestão ambiental, quanto mais proativa a organização, mais inovações tecnológicas voltadas ao meio ambiente serão encontradas. $\mathrm{O}$ atendimento a essas dimensões 
torna o processo de inovação mais sofisticado e exigente, o que requer da organização um maior esforço para atender tecnicamente esse requisito. Isso leva novas perspectivas para a gestão da inovação. Assim, pode-se dizer que a ideia da inovação sustentável é um tipo de inovação que direciona para o alcance do desenvolvimento sustentável.

Segundo Gervasoni, Rossib \& Silva (2010), a estratégia de diferenciação do produto ou serviço é baseado em diversos fatores como a produção de algo exclusivo em toda a indústria, o desenvolvimento da tecnologia utilizada na fabricação dos produtos e também a qualidade do produto. Esta diferenciação torna o cliente fiel ao produto e fortalece a marca, afastando os concorrentes.

Simultaneamente é de suma importância tornar os custos de diferenciação uma vantagem competitiva, sendo que ao reduzir os custos busca-se elevar a rentabilidade da empresa e as vendas. (GERVASONI; ROSSIB; SILVA, 2010). Desta forma é indispensável aproveitar bem os recursos existentes na organização, sejam os ativos, capacidades, processos organizacionais, atributos e informações, que ao serem controlados pela empresa permitem a implantação de ações estratégicas afim de aumentar a eficácia e por consequência a redução de custos e vantagem competitiva sustentável. (GERVASONI; ROSSIB; SILVA, 2010).

A qualidade baseada no valor é definida em termos de custo e preço, sendo um produto de qualidade aquele que apresenta um resultado ou conformidade a um preço e a um custo viável. Porém é a percepção da qualidade do cliente que é a mais importante e que por isso deve ser levada em consideração. (GERVASONI, ROSSIB, SILVA, 2010).

Bertolini et al. (2013) constatam que a questão ambiental pode proporcionar as organizações vantagem competitiva, resultando em melhora da imagem, rentabilidade e desempenho financeiro, porém para que isso se concretize, não basta a valorização dos consumidores do produto ecologicamente correto, é essencial a aquisição dos produtos pelos consumidores por um preço que proporcione lucratividade e que seja viável financeiramente a empresa.

\section{METODOLOGIA}

A pesquisa é quantitativa com abordagem exploratória, que se refere a "primeira aproximação do pesquisador com os fatos e fenômenos, confirmando ou não a real importância do problema, levantamento das informações já disponíveis podendo até mesmo indicar novas fontes." (FLEMING et al., 2005, p. 18).

O estudo foi realizado numa indústria empacotadora do setor alimentício de cereais, sendo os dados coletados a partir da adaptação do modelo Bertolini (2009).

A amostra foi definida através do método proposto por Barbetta (2008) para cálculo do tamanho mínimo de amostra, com 10\% de margem de erro. A população utilizada foi a quantidade total em quilogramas, dos três produtos mais vendidos pela industria de cereais, pipoca, amendoim e canjiquinha, durante um mês, conforme mostrado no Quado 2, abordando com questionário os consumidores que adquiriam os produtos, contando por quantidade consumida, até atingir o valor da amostra necessária, a qual foi atingido após a aplicação de 221 questionários, realizado em duas lojas de uma rede de supermercados em Cascavel-PR, que atende as classe B e C da população local. A escolha do supermercado se deu pela autorização da rede na realização da pesquisa em suas lojas, sendo que as demais redes não autorizaram. 
Quadro 2 - População e amostra.

\begin{tabular}{|l|c|c|}
\hline Produto & $\begin{array}{c}\text { População - Quantidade } \\
\text { Vendida }\end{array}$ & Amostra \\
\hline Pipoca & $2.580 \mathrm{~kg}$ & $96 \mathrm{~kg}$ \\
\hline Amendoim & $2.580 \mathrm{~kg}$ & $96 \mathrm{~kg}$ \\
\hline Canjiquinha & $1.280 \mathrm{~kg}$ & $93 \mathrm{~kg}$ \\
\hline
\end{tabular}

Fonte: Elaboração Própria.

Para Barbetta (2008), o pesquisador muitas vezes tem uma percepção sobre as variáveis estudadas sendo assim o planejamento da pesquisa deve ser de tal forma que admita testar os dados pesquisadas. Essas poderão ser verificadas utilizando-se ferramentas estatísticas denominadas testes de hipóteses.

Para o estudo foram realizados testes de independência a 10\% de significância para verificar a associação entre as variáveis pesquisadas e fatores socioeconômicos.

\subsection{Adaptação do modelo Bertolini}

O fluxograma total do modelo Bertolini (2009) completo está disposto na Figura 1.

Figura 1 - Fluxograma do modelo Bertolini

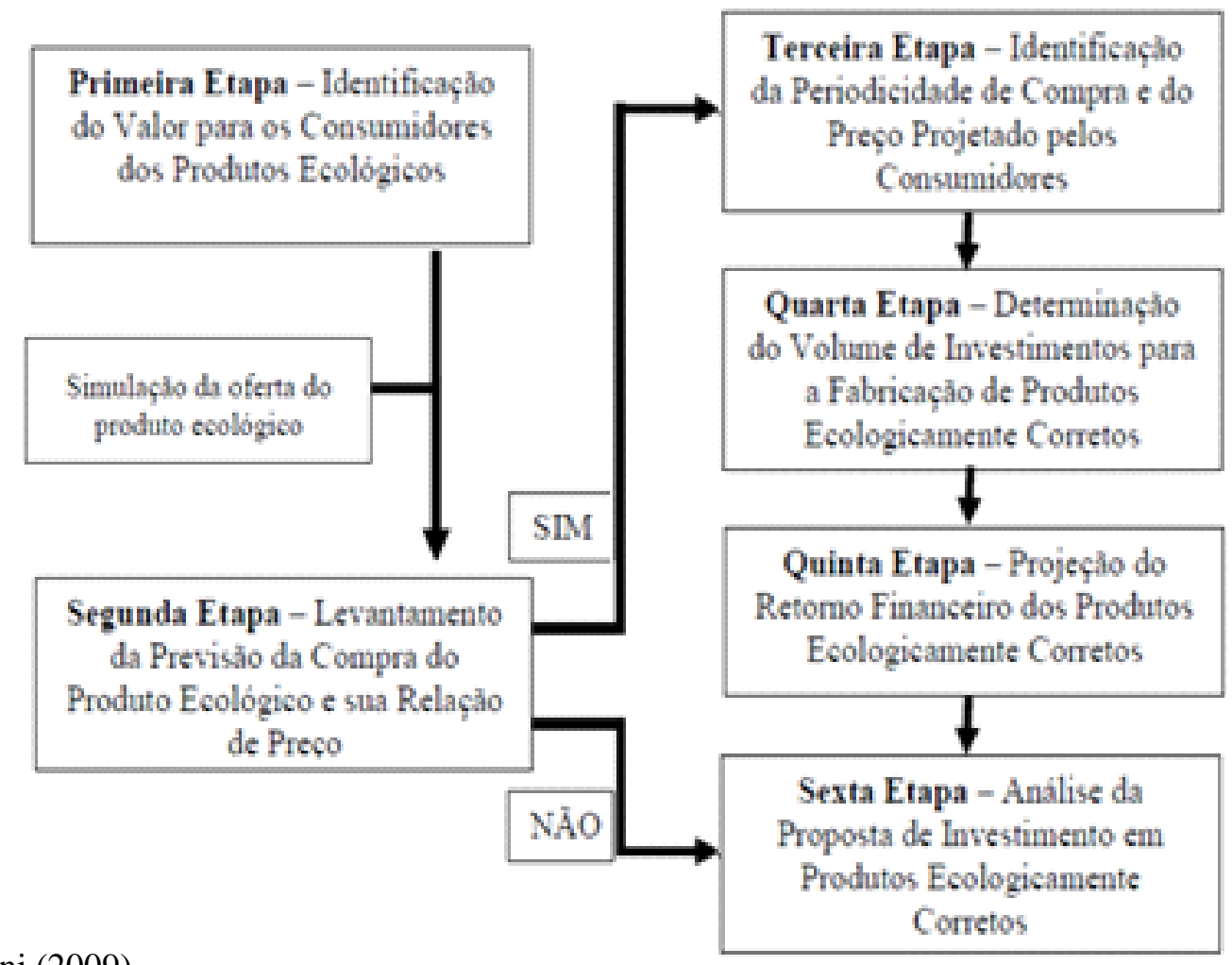

Fonte: Bertolini (2009).

Seguindo o modelo Bertolini (2009), a primeira etapa é da identificação do valor para os consumidores dos produtos ecológicos, feita através da aplicação de 221 questionários em dois supermercados de Cascavel/PR aos consumidores, composto de 12 questões relacionadas à valorização de características ambientais. Como os questionários foram feitos para três produtos (pipoca, amendoim e canjiquinha), a primeira pergunta feita para os entrevistados foi para identificar quais e que quantidade mensal desses produtos os entrevistados consomem, dessa maneira, um único consumidor pode ser 
comprador de mais de um produto e seu questionário recebe um peso de acordo com a quantidade consumida e usado para os seus respectivos produtos.

Para o presente estudo, foi feito a adaptação a partir da quarta etapa do processo do modelo Bertolini (2009), da qual se optou por fazer um caminho inverso ao que se propõe o modelo, pois não havia informações suficientes sobre os custos da matéria-prima orgânica, impossibilitando os cálculos no modelo tradicional, o que não proporciona ao final do estudo uma resposta para a viabilidade ou não do investimento, mas sim o quanto a mais cada produto pode sofrer no seu valor de venda e ainda continuar lucrativo.

\section{RESULTADOS E DISCUSSÃO}

$\mathrm{Na}$ aplicação do modelo para identificação do consumo dos produtos pesquisados, 175 dos 221 entrevistados consumiam a pipoca, e 17 dos 175 não quiseram ou preferiram não pontuar a primeira parte do questionário (primeiras sete questões), aos outros 158 entrevistados que responderam, foi solicitado que atribuíssem um grau de preferência a cada questão assinalada, distribuindo um total de 10 pontos, sendo quanto mais pontos uma questão receber, maior é o grau de preferência do consumidor por aquela característica, com a tabulação dos dados da pipoca foram levantados um total de 2817 pontos.

Seguindo as questões do questionário, ser fabricante com ações ambientais obteve um total de 511 pontos; embalagem e produto fabricado com material reciclado obteve 377 pontos; embalagem e produto reciclável recebeu 448 pontos; empresa que possui ISO 14.001 recebeu 175 pontos; produtos orgânicos recebeu 601 pontos; produtos de limpeza biodegradáveis recebeu 280 pontos; e produtos que consomem menos energia e/ou água recebeu um total de 425 pontos. Dividindo essas pontuações pela quantidade de questionários respondidos obtém a média do grau de preferência, que somam 10 pontos, conforme mostrado na Figura 2.

Figura 2 - Grau de preferência dos consumidores da pipoca

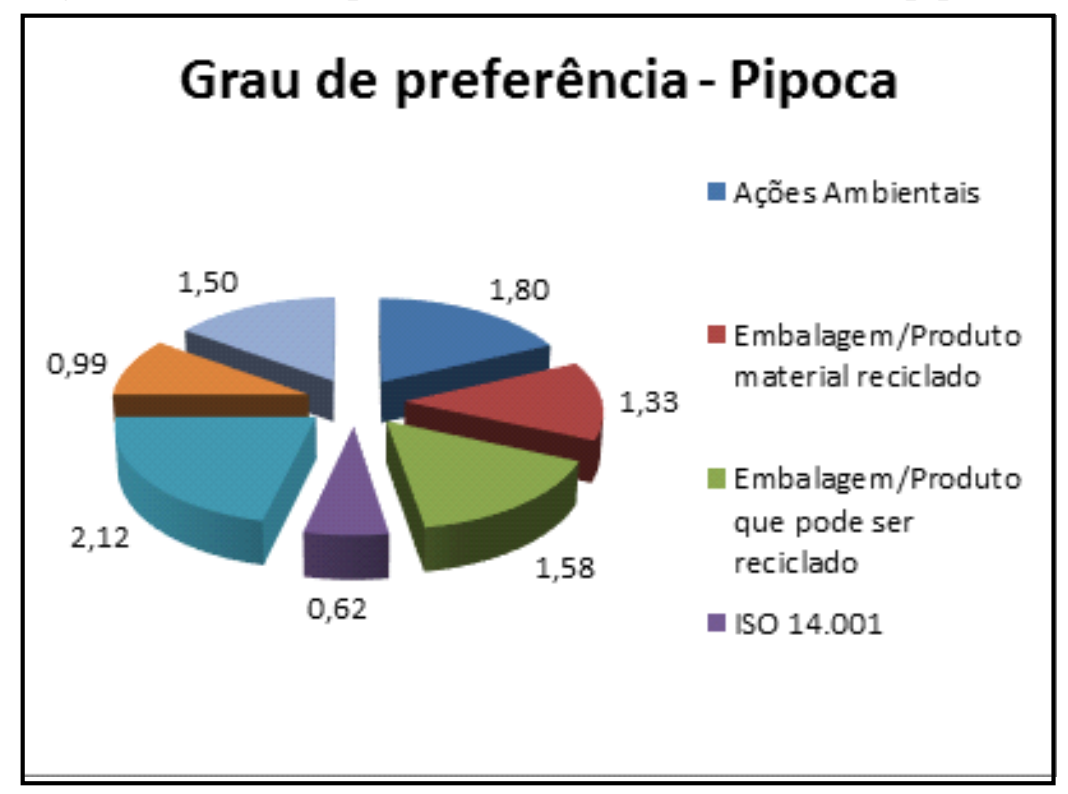

Fonte: Pesquisa de campo.

Para amendoim, 15 dos 134 entrevistados não pontuaram, então com 119 entrevistados se obteve um total de 1898 pontos - ser fabricante com ações ambientais obteve um total de 292 pontos; embalagem e produto fabricado com material reciclado obteve 219 pontos; embalagem e produto reciclável recebeu 281 pontos; empresa que possui ISO 14.001 recebeu 136 pontos; produtos orgânicos 
recebeu 385 pontos; produtos de limpeza biodegradáveis recebeu 198 pontos; e produtos que consomem menos energia e/ou água recebeu um total de 388 pontos. Dividindo essas pontuações pela quantidade de questionários respondidos obtém a média do grau de preferência, que somam 10 pontos, conforme mostrado na Figura 3.

Figura 3 - Grau de preferência dos consumidores do amendoim

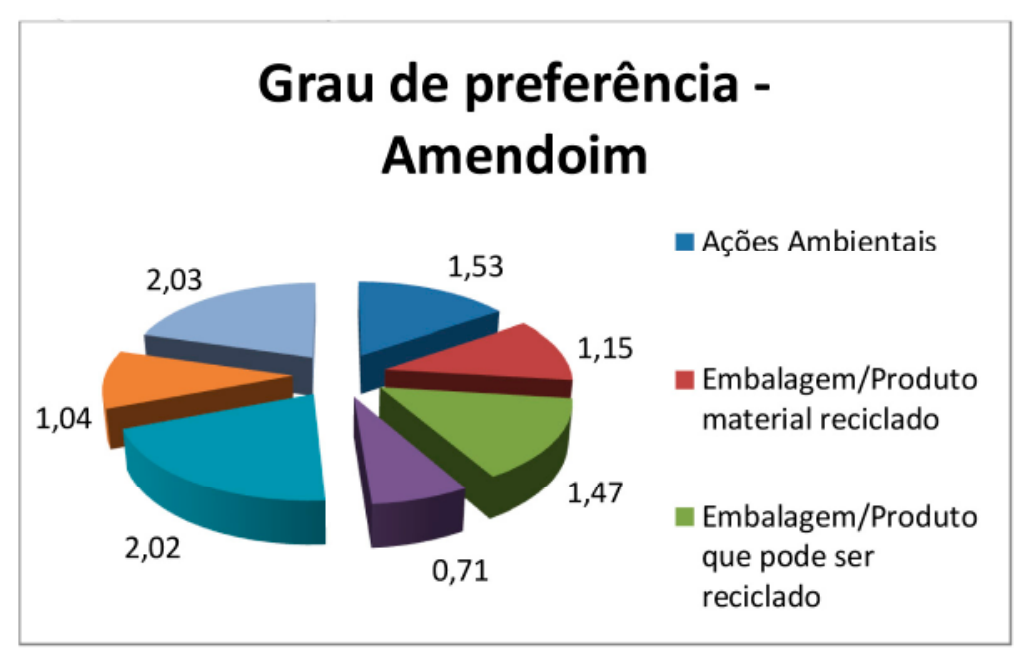

Fonte: Pesquisa de campo.

Para canjiquinha, 17 dos 132 entrevistados não pontuaram, então com 115 entrevistados se obteve um total de 1557 pontos - ser fabricante com ações ambientais obteve um total de 264 pontos; embalagem e produto fabricado com material reciclado obteve 206 pontos; embalagem e produto reciclável recebeu 260 pontos; empresa que possui ISO 14.001 recebeu 130 pontos; produtos orgânicos recebeu 324 pontos; produtos de limpeza biodegradáveis recebeu 121 pontos; e produtos que consomem menos energia e/ou água recebeu um total de 251 pontos. Dividindo essas pontuações pela quantidade de questionários respondidos obtém a média do grau de preferência, que somam 10 pontos, conforme mostrado na Figura 4.

Figura 4 - Grau de preferência dos consumidores da canjiquinha

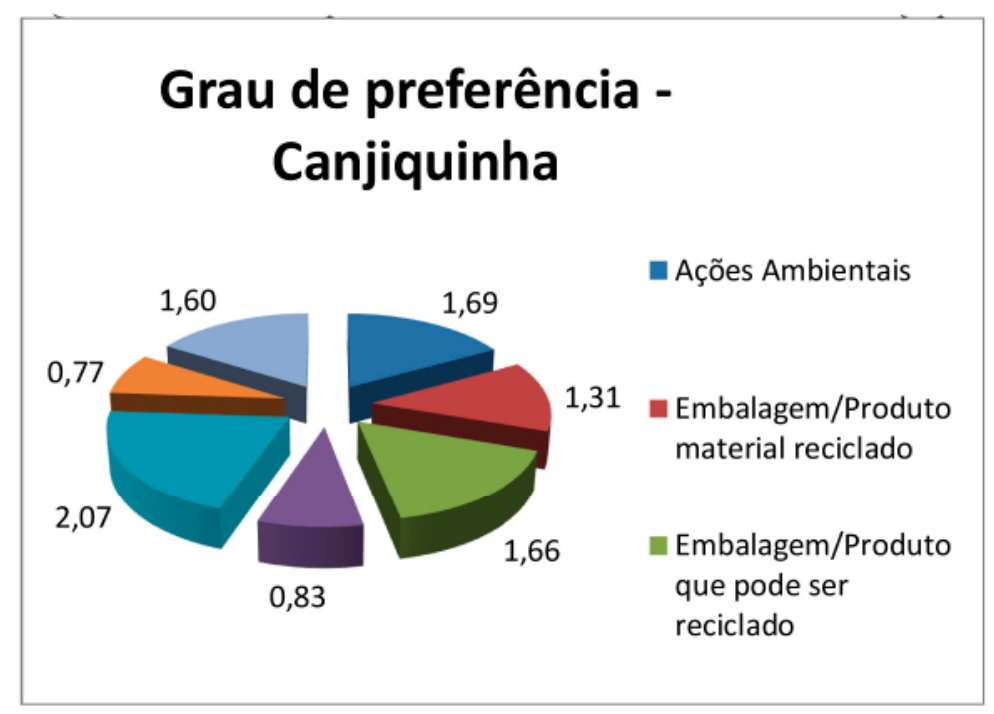

Fonte: Pesquisa de campo. 
É possível identificar que, apesar de possuir certa homogeneidade, a característica mais valorizada pelos consumidores é a dos produtos ecológicos, sendo essa a característica base do estudo.

As questões 8 a 12 do modelo Bertolini (2009) buscou a identificação do perfil dos consumidores entrevistados, a Figura 5 mostra a questão 8 - o grau de escolaridade dos entrevistados, tendo a maioria (44\%) dos entrevistados o ensino médio completo.

Figura 5 - Grau de escolaridade

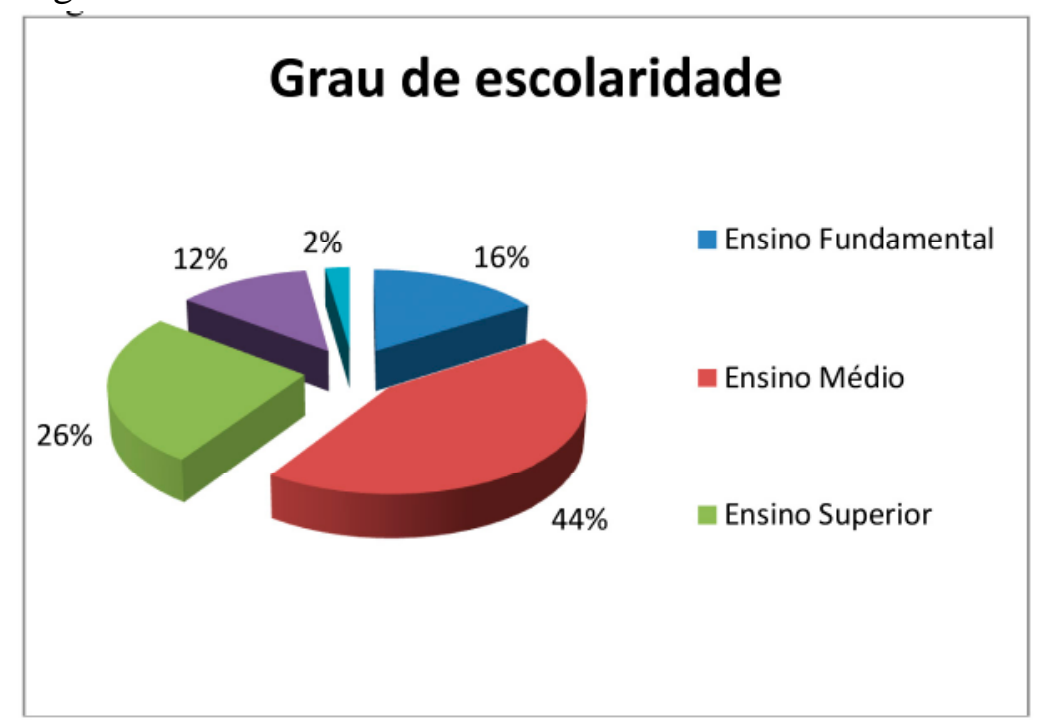

Fonte: Pesquisa de campo.

A figura 6 se refere à questão 9, que demonstra os salários mensais dos entrevistados, observase que há predominância (60\%) de entrevistados com mais de um a cinco salários mínimos.

Figura 6 - Salário

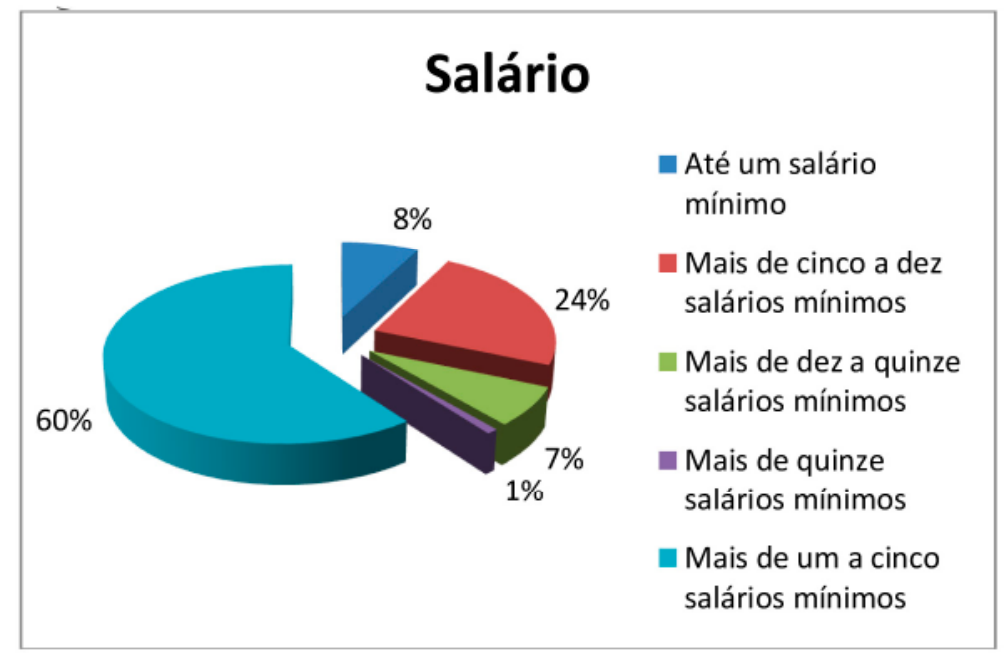

Fonte: Pesquisa de campo.

A Figura 7 mostra a relação de idade dos entrevistados, que foi a questão 10 feita a eles e identifica a maioria como pessoas de 35 a 49 anos (41\%) e acima de 50 anos (34\%). 
Figura 7 - Idade

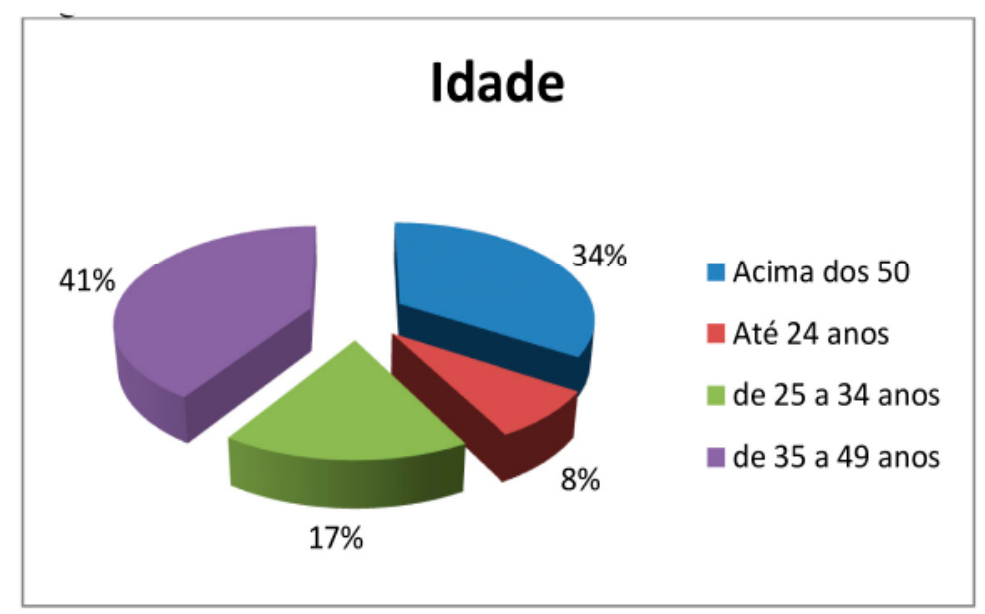

Fonte: Pesquisa de campo.

A questão 11 se refere aos bairros de Cascavel onde os entrevistados moram e suas respectivas quantidades de pessoas - de acordo com os pesos de consumo de cada um, assim, os bairros e quantidades de pessoas que consomem cada produto são demonstrados nos quadros 3 , 4 e 5 .

Quadro 3 - Bairros dos consumidores: Pipoca

\begin{tabular}{|c|c|c|c|c|c|c|c|}
\hline 14 de Novembro & 20 & Guarujá & 12 & outro município & 3 & Tropical & 6 \\
\hline Aclimação & 12 & Interlagos & 3 & Pacaembu & 3 & Vila Tolentino & 6 \\
\hline Alto Alegre & 5 & Itapuã & 2 & Paranaguá & 3 & & \\
\hline Angra dos Reis & 2 & Jardim Alvorada & 4 & Parque São Paulo & 8,5 & & \\
\hline Brasmadeira & 1 & $\begin{array}{l}\text { Jardim Nova } \\
\text { Iorque }\end{array}$ & 3 & Parque Verde & 6 & & \\
\hline Cancelli & 1 & Jardim União & 5 & Paulo Godoy & 1 & & \\
\hline Centro & 32 & Lago I & 1 & \begin{tabular}{|l|} 
Pioneiros \\
Catarinenses
\end{tabular} & 13,5 & & \\
\hline Ciro Nardi & 6 & Maria Luiza & 6 & Quebec & 7 & & \\
\hline Consolata & 1 & Montreal & 1 & Roma & 1 & & \\
\hline Country & 3 & Morumbi & 3 & Santa Cruz & 10 & & \\
\hline Esmeralda & 5 & Neva & 93 & Santa Felicidade & 4 & & \\
\hline Esplanada & 1 & Nova Cidade & 2 & Santo Onofre & 1 & & \\
\hline Faculdade & 1 & Nova Laranjeiras & 1 & São Cristóvão & 11 & & \\
\hline
\end{tabular}

Fonte: Pesquisa de campo

Quadro 4 - Bairros dos consumidores: Amendoim

\begin{tabular}{|l|l|l|l|l|l|l|l|}
\hline 14 de Novembro & 14 & Guarujá & 7,33 & Outro município & 3,5 & Tropical & 3 \\
\hline Aclimação & 0 & Interlagos & 2 & Pacaembu & 2 & $\begin{array}{l}\text { Vila } \\
\text { Tolentino }\end{array}$ & 6 \\
\hline Alto Alegre & 2 & Itapuã & 0 & Paranaguá & 3 & & \\
\hline Angra dos Reis & 2 & Jardim Alvorada & 1 & Parque São Paulo & 9,5 & & \\
\hline Brasmadeira & 0 & Jardim Nova & 0 & Parque Verde & 4 & & \\
Cancelli & 1 & Jardim União & 4 & Paulo Godoy & 0 & & \\
\hline
\end{tabular}




\begin{tabular}{|l|c|l|c|l|c|l|}
\hline Centro & 16 & Lago I & 1 & $\begin{array}{l}\text { Pioneiros } \\
\text { Catarinenses }\end{array}$ & 9,5 & \\
\hline Ciro Nardi & 2 & Maria Luiza & 3 & Quebec & 3 & \\
\hline Consolata & 1 & Montreal & 0 & Roma & 1 & \\
\hline Country & 4 & Morumbi & 6 & Santa Cruz & 6,3 & \\
\hline Esmeralda & 1,5 & Neva & 77 & Santa Felicidade & 5 & \\
\hline Esplanada & 1 & Nova Cidade & 0 & Santo Onofre & 0 & \\
\hline Faculdade & 1 & Nova Laranjeiras & 1 & São Cristóvão & 7,5 & \\
\hline
\end{tabular}

Fonte: Pesquisa de campo.

Quadro 5 - Bairros dos consumidores: Canjiquinha

\begin{tabular}{|l|l|l|l|l|l|l|l|}
\hline Brasmadeira & 11 & Guarujá & 4,17 & Outro município & 3 & Tropical & 3 \\
\hline Aclimação & 2 & Interlagos & 5 & Pacaembu & 1 & Vila Tolentino & 4 \\
\hline Alto Alegre & 6 & Itapuã & 1 & Paranaguá & 3 & Cascavel Velho & 1 \\
\hline Angra dos Reis & 1 & Jardim Alvorada & 1 & Parque São Paulo & 20 & Jardim Itália & 2 \\
\hline Brasmadeira & 0 & $\begin{array}{l}\text { Jardim Nova } \\
\text { Iorque }\end{array}$ & 0 & Parque Verde & 1 & Turispark & 2 \\
\hline Cancelli & 4 & Jardim União & 1 & Paulo Godoy & 1 & Universitário & 1 \\
\hline Centro & 22 & Lago I & 1 & $\begin{array}{l}\text { Pioneiros } \\
\text { Catarinenses }\end{array}$ & 16 & & \\
\hline Ciro Nardi & 0 & Maria Luiza & 1 & Quebec & 5 & & \\
\hline Consolata & 2 & Montreal & 0 & Roma & 1 & & \\
\hline Country & 2 & Morumbi & 2 & Santa Cruz & 3,33 & & \\
\hline Esmeralda & 1,08 & Neva & 40 & Santa Felicidade & 3 & & \\
\hline Esplanada & 0 & Nova Cidade & 2 & Santo Onofre & 0 & & \\
\hline Faculdade & 0 & Nova Laranjeiras & 1 & São Cristóvão & 5,5 & & \\
\hline
\end{tabular}

Fonte: Pesquisa de campo

A questão 12 se refere ao sexo dos entrevistados, conforme disposto na Figura 8, podendo identificar que a maioria $(65 \%)$ dos entrevistados foram do sexo feminino.

Figura $8-$ Sexo

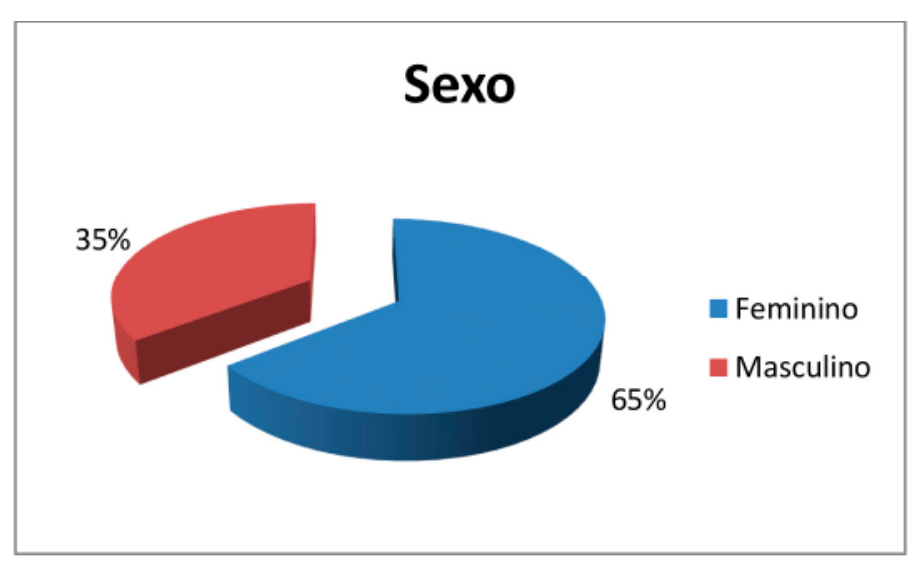

Fonte: Pesquisa de campo. 
De posse desses dados, foram desenvolvidos testes Qui-Quadrado para independência, a 10\% de significância, do perfil socioeconômico em relação a importância dada aos produtos orgânicos pelos consumidores, o ponto principal do estudo, assim, se verificou o gênero dos entrevistados não dão valor a produtos ecológicos, pois o p-valor se mostrou maior que 0,1, como mostra o Quadro 6.

Quadro 6 - Gênero e importância dada a orgânicos

\begin{tabular}{|cc|}
\hline Teste Qui-Quadrado Gênero & \\
\hline Estatística X ${ }^{2}$ & 2,320938313 \\
Graus de Liberdade & 4 \\
P-Valor & 0,67695955 \\
\hline
\end{tabular}

Fonte: Pesquisa

Da mesma forma, as variáveis sobre renda dos entrevistados em comparação com a preferência por produto orgânico não obtiveram relação, conforme demonstrado no quadro 7 .

Quadro 7 - Renda e preferência por produto orgânico

\begin{tabular}{|cc|}
\hline Teste Qui-Quadrado Renda & \\
Estatística X ${ }^{2}$ & 9,32796759 \\
Graus de Liberdade & 6 \\
P-Valor & 0,155956124 \\
\hline
\end{tabular}

Fonte: Pesquisa

O mesmo resultado também foi levantado pela variável grau de escolaridade, mostrada no Quadro 8, o que não é possível afirmar que a escolaridade tem relação com a preferência de consumir produtos orgânicos.

Quadro 8 - Grau de escolaridade e preferência por produto orgânico

\begin{tabular}{|cc|}
\hline Teste Qui-Quadrado Grau de escolaridade & \\
\hline Estatística X ${ }^{2}$ & 1,987311642 \\
Graus de Liberdade & 6 \\
P-Valor & 0,92086184 \\
\hline
\end{tabular}

Fonte: Pesquisa

Já na variável faixa etária em comparação com a preferência por produto orgânico obteve-se relação, com p-valor resultante inferior a 0,10 , mostrando que a preferência por produto orgânico muda conforme a faixa de etária, demonstrado no Quadro 9.

Quadro 9 - Faixa etária e preferência por produto orgânico

\begin{tabular}{|cc|}
\hline Teste Qui-Quadrado Faixa etária & 15,16136965 \\
Estatística X ${ }^{2}$ & 6 \\
Graus de Liberdade & 0,019038078 \\
\hline
\end{tabular}

Fonte: Pesquisa

Sendo assim, os entrevistados puderam optar pela decisão de compra do produto orgânico, que, conforme o modelo Bertolini (2009), indicam a preferência dos compradores, mostrado no Quadro 10. 
Quadro 10 - Preferência de compra

\begin{tabular}{|c|c|}
\hline Resultado & Preferência de compra \\
\hline Zero a 0,99 & Não comprarão o produto. \\
\hline 1 a 2,99 & Comprarão o produto, desde que seu preço seja inferior aos demais. \\
\hline 3 a 4,99 & Comprarão o produto, desde que seu preço seja igual aos demais. \\
\hline 5 a 6,99 & Comprarão o produto e pagarão um pouco a mais por ele. \\
\hline 7 a 8 & Comprarão o produto independente de seu preço. \\
\hline
\end{tabular}

Fonte: Bertolini (2009).

Fazendo os cálculos dos entrevistados, conforme o modelo de Bertolini (2009), obteve a pontuação de 5,57, da qual indica que os consumidores comprarão o produto e pagarão um pouco a mais por ele, demonstrado no Quadro 11.

Quadro 11 - Pontuação dos consumidores

\begin{tabular}{|c|c|c|}
\hline Quantidade de respostas & Pontuação & (a X b) Resultado \\
\hline A- 17 & 2 & 34 \\
\hline B- 87 & 4 & 348 \\
\hline C- 43 & 6 & 258 \\
\hline D- 74 & 8 & 592 \\
\hline \multicolumn{2}{|c|}{ (c) Soma dos resultados } & 1232 \\
\hline \multicolumn{2}{|c|}{ (d) número de entrevistados } & 221 \\
\hline \multicolumn{2}{|c|}{ (e c c/d) Resultado da preferência } \\
\hline
\end{tabular}

Fonte: Pesquisa.

O próximo passo foi calcular a viabilidade de se fabricar os produtos orgânicos e optou-se por fazer um caminho inverso do modelo Bertolini (2009), pois, conforme mencionado na metodologia, não há informações suficientes sobre os custos da matéria-prima orgânica, impossibilitando os cálculos no modelo tradicional, assim sendo, os resultados que foram obtidos é o de acréscimo máximo unitário no preço dos produtos para que se mantenham lucrativos, em vez de analisar se há viabilidade. Isso permite à empresa obter informações para sua precificação no mercado.

O sobre valor que os consumidores estão dispostos a pagar pelos produtos orgânicos, que para pipoca foi levantado uma aceitação de aumento de $16,51 \%$ no valor do produto normal ( $\mathrm{R} \$ 1,89$ - média de várias marcas de pipoca), chegando-se então ao preço projetado de $\mathrm{R} \$ 2,20$ ( $\mathrm{R} \$ 1,89$ x 16,51\%), podendo a empresa aumentar seus custos em $\mathrm{R} \$ 0,31(\mathrm{R} \$ 2,20-\mathrm{R} \$ 1,89)$ centavos à unidade. $\mathrm{O}$ consumo por mês, a quantidade vendida fornecida pela empresa, é de 5.160 pacotes de $500 \mathrm{~g}$, atingindo uma projeção da valorização total periódica (P.V.T.p.) de $\mathrm{R} \$ 99,64$, dividindo esse valor com a quantidade vendida, temos a projeção da valorização unitária de $\mathrm{R} \$ 0,02$, dessa maneira, chegamos à conclusão que os custos adicionais máximos que a empresa pode acrescentar por unidade de produto vendido pode se chegar a até $\mathrm{R} \$ 0,29(\mathrm{R} \$ 0,31-\mathrm{R} \$ 0,02)$.

Para amendoim foi levantado uma aceitação de aumento de $14,54 \%$ no valor do produto normal ( $\mathrm{R} \$ 3,42$ - média de várias marcas de amendoim), chegando-se então ao preço projetado de $\mathrm{R} \$ 3,92$ $(\mathrm{R} \$ 1,89$ x 14,54\%), podendo a empresa aumentar seus custos em $\mathrm{R} \$ 0,50(\mathrm{R} \$ 3,92-\mathrm{R} \$ 3,42)$ centavos à unidade. O consumo por mês, a quantidade vendida fornecido pela empresa, é de também 5.160 pacotes de 500g, atingindo uma projeção da valorização total periódica (P.V.T.p.) de R\$99,64, dividindo esse valor com a quantidade vendida, temos a projeção da valorização unitária de $\mathrm{R} \$ 0,02$, dessa maneira, chegamos à conclusão que os custos adicionais máximos que a empresa pode acrescentar por unidade de produto vendido pode se chegar a até $\mathrm{R} \$ 0,48(\mathrm{R} \$ 0,50-\mathrm{R} \$ 0,02)$. 
Já para canjiquinha foi levantado uma aceitação de aumento de $24,32 \%$ no valor do produto normal ( $\mathrm{R} \$ 1,43$ - média de várias marcas de amendoim), chegando-se então ao preço projetado de $\mathrm{R} \$ 1,78$ ( $\mathrm{R} \$ 1,43$ x 24,32\%), podendo a empresa aumentar seus custos em $\mathrm{R} \$ 0,35$ ( $\mathrm{R} \$ 1,78-\mathrm{R} \$ 1,43$ ) centavos à unidade. $\mathrm{O}$ consumo por mês, a quantidade vendida fornecida pela empresa, é de 2.560 pacotes de $500 \mathrm{~g}$, atingindo uma projeção da valorização total periódica (P.V.T.p.) de R $\$ 99,64$, dividindo esse valor com a quantidade vendida, temos a projeção da valorização unitária de $\mathrm{R} \$ 0,04$, dessa maneira, chegamos à conclusão que os custos adicionais máximos que a empresa pode acrescentar por unidade de produto vendido pode se chegar a até $\mathrm{R} \$ 0,31(\mathrm{R} \$ 0,35-\mathrm{R} \$ 0,04)$.

\section{CONCLUSÃo}

O modelo Bertolini (2009) se mostrou bem adequado para o estudo, pois foi possível a partir dele, levantar as informações necessárias para um estudo da disposição, ou seja, por um lado foi possível levantar e identificar a percepção e opinião dos consumidores finais sobre suas preferências e sobre a mudança dos produtos em questão para se tornarem ecológicas, o quanto estão dispostos a pagar a mais por essa mudança, por outro lado, também foi possível analisar a partir do ponto de vista da organização e suas necessidades de gerar valor, tanto para os consumidores e a sociedade, oferecendo produtos ecologicamente corretos, quanto para a empresa e seus sócios em relação aos retornos financeiros e estratégicos.

Dessa maneira, após a análise dos dados, identificou-se que os consumidores possuem um maior grau de preferência em relação aos produtos orgânicos, sendo essa a variável mais pontuadora para todos os entrevistados compradores de pipoca, amendoim e canjiquinha, o que justifica a necessidade do estudo em questão, a fim de auxiliar as empresas nas suas tomadas de decisão. Os testes de quiquadrado só deram significativo para faixa etária.

Como contribuição do estudo, a partir da adaptação do modelo, foi possível levantar, ao invés da viabilidade de investimentos o que propõe o modelo Bertolini (2009), quanto de aumento no custo unitário que cada um dos produtos estudados, a pipoca, amendoim e canjiquinha suportam para se manterem lucrativas. Como limitação do estudo, pode-se indicar a possibilidade de coleta de dados que foram dois supermercados, isso pela não autorização de realização em outros.

Com a análise, foi identificado que para o produto pipoca, a empresa pode ter um aumento no custo em até $\mathrm{R} \$ 0,29$ centavos e manter-se lucrativa, já para o produto amendoim, o custo adicional pode ser de até $\mathrm{R} \$ 0,48$ centavos e ainda ser viável, por fim, para o produto canjiquinha, o custo pode ser de até $\mathrm{R} \$ 0,31$ centavos a mais e ainda dar retornos para a empresa.

Há de se notar que os consumidores que não pagariam a mais por um produto orgânico não são de forma alguma um público que seria eliminado como consumidores da empresa, pois a inclusão dos produtos orgânicos não acarretaria a retiradas dos produtos normais das prateleiras, assim, a introdução dos produtos orgânicos aumentaria o valor agregado que a organização poderá mostrar para os consumidores. Tais resultados corroboram como contribuições teóricas para o campo do comportamento do consumidor.

Por fim, sugere-se que a pesquisa em relação ao grau de preferência dos consumidores possa ser aplicada para outras populações de outras regiões e também aplicá-las à população da região a fim de identificar se o grau de preferência por produtos orgânicos e a disposição de valor que se comprometem a pagar por tais produtos virão a se tornar ainda mais importantes, pois caso a empresa não tenha condições de implantar uma produção de produtos orgânicos neste momento, num futuro próximo, com outras pesquisas em relação ao valor que esse tipo de produto têm para o cliente, possa vir a se tornar viável tanto ecologicamente quanto financeiramente. 


\section{REFERÊNCIAS}

AGUIAR, L. K.; CALEMAN, S. M. Q. Sustainability: who is driving it? Desafio Online, Campo Grande, MS, v.1, n.2, 1-24, maio/ago. 2013.

ALVES, William Ferreira; ARAÚJO, Aracy Alves; SILVA, Claudionor Ribeiro. Percepção ambiental dos moradores de Uberlândia sobre a estação ecológica do Panga - uma visão sob a ótica da economia do meio ambiente. Revista Ibero-Americana de Ciências Ambientais, Aracaju, v. 8 n. 4, 2017.

BARBETTA, P. A. Estatística aplicada às ciências sociais. Florianópolos, SC: UFSC, 2008.

BERTOLINI, G. R. Modelo para a identificação do volume de Investimentos na fabricação de produtos Ecologicamente corretos. 2009. 253 f. Tese (Doutorado em Engenharia de Produção) Programa de Pós-Graduação em Engenharia de Produção- Universidade Federal de Santa Catarina, Florianópolis, SC, Brasil, 2009.

BERTOLINI, G. R. et al. A viabilidade financeira no desenvolvimento de produtos ecológicos valorizados pelos consumidores. Revista de Gestão e Projetos, São Paulo, v.3, n.3, p. 01-29, set./dez. 2013.

BERTOLINI, G. R.; LEZANA, Á. G.;ROJO, C. A. Modelo de análise de investimentos para fabricação de produtos ecologicamente corretos. Gestão \& Produção, São Carlos, SP, v.19, n.3, p. 575$588,2012$.

CERDA URRUTIA, Arcadio Alberto et al. Preferencias e disposição em pagar por uva de mesa orgânica na região de Maule, Chile. Rev. Bras. Frutic., Jaboticabal, SP, v. 33, n. 3, p. 784-790, Sept. 2011.

CESCHIM, G.; MARCHETTI, R. Z. O Comportamento inovador entre consumidores de produtos orgânicos: uma abordagem qualitativa. In: ENCONTRO DA ASSOCIAÇÃO NACIONAL DE PÓS-GRADUAÇÃO E PESQUISA EM ADMINISTRAÇÃO, 2009, São Paulo. Anais [...]. São Paulo: EnANPAD, 2009.

CLEMENTIN, N. Orgânicos apresentam expansão na produção e interesse de cosumo. Jan.2014. Disponível em:<http://g1.globo.com/sao-paulo/sao-jose-do-rio-preto-aracatuba/noticia/2014/01/ organicos-apresentam-expansao-na-producao-e-interesse-de-consumo.html>. Acesso em: 07 jul. 2014.

FLEMING, S. F. et al. Manual para elaboração de trabalhos científicos, redação oficial e comercial. Cascavel, PR: Coluna do Saber, 2005.

FREITAS, C. A. et al. A. Um estudo preliminar sobre a viabilidade do sistema de produção orgânico baseado em suas características econômicas. 2005. Disponível em: http://www.sober.org. br/palestra/2/733.pdf. Acesso em: 07 jul. 2014. 
GERVASONI, V. C.;ROSSIB, G. B.; SILVA, D. Gestão de custos e despesas e a estratégia competitiva de diferenciação - setor autopeças. Revista Brasileira de Estratégia, Curitiba,PR, v.3, n.3, p.217-230, 2010.

HEINZEN, D. A. M.;MATTOS, A. P. M. N.;CAMPOS, L. M. S.;PALADINI, E. P.Estudo da viabilidade de produto inovador VERDE para o mercado consumidor comum. Revista Gestão Organizacional, Chapecó, SC, v. 4, n. 2, p. 233-251, 2011.

INSTRUÇÃO NORMATIVA nº46, de 6 de outubro de 2011. Ministério da Agricultura, Pecuária e Abastecimento.

LOMBARDI, M. S.; MOORI, R. G.; SATO, G. S. Um estudo exploratório dos fatores relevantes na Decisão de compra de produtos orgânicos. Revista de Administração Mackenzie, São Paulo, v.5, n.1, p. 13-34, 2004.

LOPES, V. N.; PACAGNAN, M. N. Marketing verde e práticas socioambientais nas indústrias do Paraná. RAUSP, São Paulo, v.49, n.1, p. 116-128, jan./fev./mar. 2014.

ROMEIRO, Ademar Ribeiro. Desenvolvimento sustentável: uma perspectiva econômico-ecológica.

Estud. av., São Paulo, v. 26, n.74, p. 65-92, 2012.

ROSSETTI, J. P. Introdução a economia. São Paulo: Atlas, 1997.

SAMPAIO, D. O. et al. Uma análise da produção acadêmica brasileira sobre o comportamento do consumidor de alimento orgânico entre 1997 a 2011. REAd. Revista Eletrônica de Administração, Porto Alegre, RS, v.19, n.3, p.620-64, 2013.

SILVA, R. M. S. et al. Disposição a pagar e comportamento ecológico dos consumidores. Gestão \& Sociedade, Belo Horizonte, MG, v.13, n.36, p.3090-3113. 2019.

TAMBOSI, Silvana Silva Vieira et al. Consciência ambiental, hábitos de consumo sustentável e intenção de compra de produtos ecológicos de alunos de uma IES de Santa Catarina. Revista Eletrônica de Administração e Turismo - ReAT, Pelotas, RG, v. 5, n. 3, 2014.

TILMAN, D.;CASSMAN, K. G.;MATSON, P. A.;NAYLOR, R.; POLASKY, S. Agricultural sustainability and intensive production practices. Nature, v.418, p. 671-677, ago. 2002.

TREVIZAN, S. D.; CASEMIRO, A. D. Consumidores de produtos orgânicos em Vitória da Conquista. In: SECOND INTERNATIONAL WORKSHOP ADVANCES IN CLEANER PRODUCTION, São Paulo, 20 a 22 de Maio de 2009. 